\title{
Swapping Exchange and Spin-Orbit Coupling in 2D van der Waals Heterostructures
}

\author{
Klaus Zollner®, ${ }^{1, *}$ Martin Gmitra, ${ }^{2}$ and Jaroslav Fabian $\oplus^{1}$ \\ ${ }^{1}$ Institute for Theoretical Physics, University of Regensburg, 93053 Regensburg, Germany \\ ${ }^{2}$ Institute of Physics, P. J. Šafárik University in Košice, 04001 Košice, Slovakia
}

(Received 4 June 2020; accepted 24 September 2020; published 3 November 2020)

\begin{abstract}
The concept of swapping the two most important spin interactions-exchange and spin-orbit couplingis proposed based on two-dimensional multilayer van der Waals heterostructures. Specifically, we show by performing realistic ab initio simulations, that a single device consisting of a bilayer graphene sandwiched by a $2 \mathrm{D}$ ferromagnet $\mathrm{Cr}_{2} \mathrm{Ge}_{2} \mathrm{Te}_{6}$ (CGT) and a monolayer $\mathrm{WS}_{2}$, is able not only to generate, but also to swap the two interactions. The highly efficient swapping is enabled by the interplay of gate-dependent layer polarization in bilayer graphene and short-range spin-orbit and exchange proximity effects affecting only the layers in contact with the sandwiching materials. We call these structures ex-so-tic, for supplying either exchange (ex) or spin-orbit (so) coupling in a single device, by gating. Such bifunctional devices demonstrate the potential of van der Waals spintronics engineering using 2D crystal multilayers.
\end{abstract}

DOI: 10.1103/PhysRevLett.125.196402

Introduction.-Novel 2D van der Waals heterostructures give a strong push to spintronics [1-3] which requires electrical control of spin interactions to realize devices such as spin transistors [4-8]. The past years have seen impressive progress in tuning spin-orbit and exchange couplings individually [9-21]. A particularly suitable platform for gating proximity effects is based on bilayer graphene (BLG). It was proposed that spin-orbit coupling in BLG can be turned on and off on demand [22,23], as recently demonstrated [24]. Similar effects have been predicted for ferromagnetic encapsulation [25-27]. The next milestone would be a transport demonstration with sensitivity to the spin polarization of the bands, or the spinorbit torque in the proximity setup [28-30].

In this Letter we present a device structure which is capable of not only creating the two most important spin interactions - spin-orbit and exchange couplings - in an electronic system, but also swapping them on demand by an applied electric field. Being able to swap two different spin (or other effective) interactions by gating is a striking thought, without precedence in the realm of conventional materials. While proximity spin-orbit coupling preserves time-reversal symmetry, and leads to such phenomena as topological quantum spin Hall effect [31] or giant spin relaxation anisotropy [14,32-34], proximity exchange coupling breaks time reversal symmetry and renders a nominally nonmagnetic electronic system effectively magnetic [35]. Swapping the two interactions provides a reversible route between time-reversal symmetric and magnetic physics.

Our choice of the electronic platform for swapping the spin interactions is BLG, which is nicely suited for proximity-based devices by providing two coupled surfaces. Via layer polarization, which locks a given layer to an electronic band (or set of bands), transport properties can be strongly influenced by the environment. We use a CGT/BLG/WS $\mathrm{W}_{2}$ heterostructure, with monoloayer $\mathrm{Cr}_{2} \mathrm{Ge}_{2} \mathrm{Te}_{6}(\mathrm{CGT})$ providing a strong proximity exchange effect to the bottom layer of $\mathrm{BLG}$, and $\mathrm{WS}_{2}$ to induce strong spin-orbit coupling to the top layer, see Fig. 1.

Gating can swap spin-orbit and exchange couplings, as illustrated in Fig. 1. The applied electric field changes the layer polarization, which changes the layer-band assignment, thereby swapping the proximity exchange and spinorbit couplings. This intuitive picture is supported below by realistic density functional theory (DFT) simulations and phenomenological modeling, predicting quantitatively the behavior of the ex-so-tic heterostructures in the presence of a transverse electric field.

Swapping spin-orbit and exchange coupling by gate.We consider a supercell stack containing BLG sandwiched between a monolayer $\mathrm{WS}_{2}$ and a monolayer ferromagnetic CGT with an out-of-plane magnetization, as depicted in Fig. 1. We calculate the electronic states for this structure using DFT, see Supplemental Material [36]. The band structure along selected high-symmetry lines containing the $K$ point is shown in Fig. 2. The magnetization-from CGT -as well as the strong spin-orbit coupling-from the transition-metal dichalcogenide (TMDC) - are manifested by the spin polarization of the bands. Inside the semiconductor band gap, there are well preserved parabolic electronic states of BLG. States relevant for transport form the four low-energy bands close to the Fermi level. It is these four bands that are at the focus of this work.

BLG per se has no band gap, has tiny spin-orbit coupling (on the order of tens of $\mu \mathrm{eVs}$ [37]), and has no magnetic exchange coupling. But sandwiched by the two monolayers, both orbital and spin properties of the low-energy 


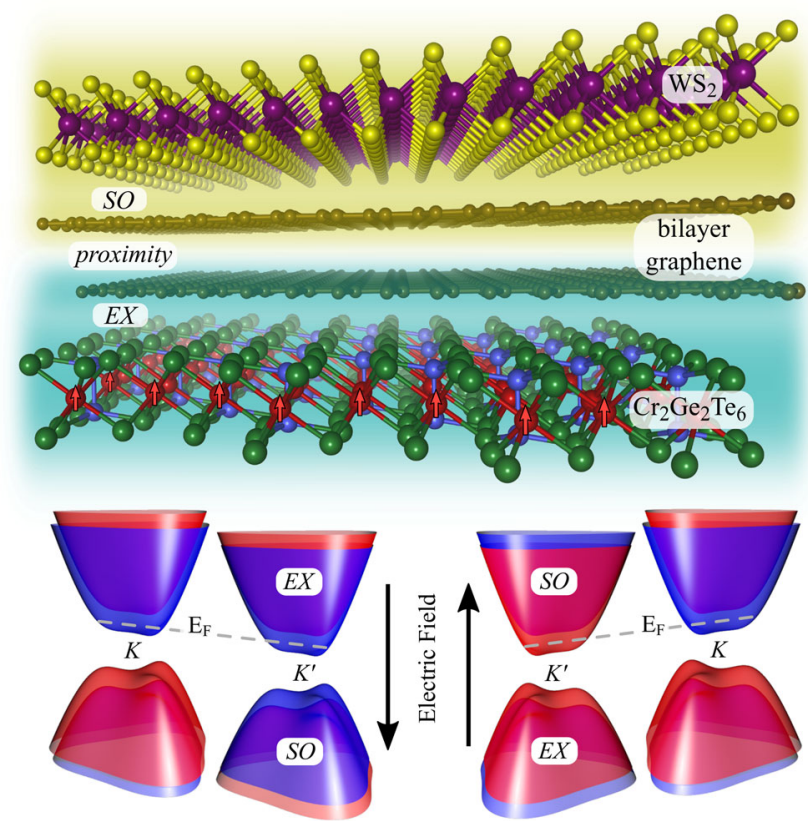

FIG. 1. Ex-so-tic van der Waals heterostructure. Top: BLG sandwiched between a monolayer TMDC (such as $\mathrm{WS}_{2}$ or $\mathrm{MoSe}_{2}$ ) and a monolayer ferromagnetic semiconductor (such as CGT or $\mathrm{CrI}_{3}$ ). The magnetization of the ferromagnet is indicated by the arrows. The top layer of BLG is proximitized by the TMDC, acquiring a giant spin-orbit coupling, while the bottom layer of the BLG is proximitized by the ferromagnet, acquiring an exchange coupling. Bottom: Electric tunability of the low-energy bands at $K$ and $K^{\prime}$. The colors red and blue indicate out-of-plane spin. For a fixed Fermi level $E_{F}$ (here in the conduction band), the Dirac electrons experience either exchange or spin-orbit coupling, depending on the electric field.

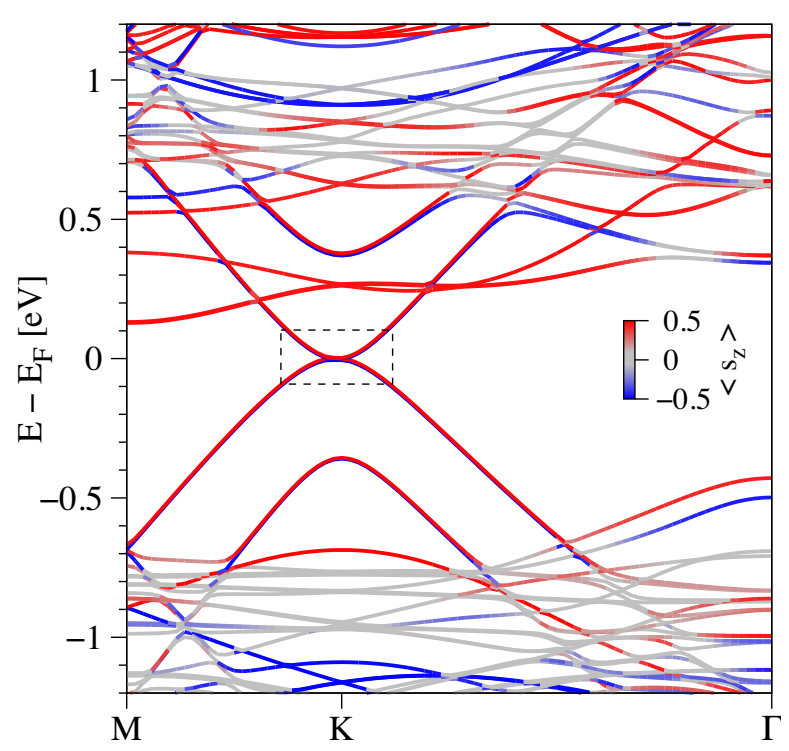

FIG. 2. Calculated electronic band structure of a $\mathrm{WS}_{2} / \mathrm{BLG} /$ CGT stack along the high symmetry path $\mathrm{M}-\mathrm{K}-\Gamma$. The color of bands (red and blue) corresponds to the $s_{z}$ spin expectation value. We focus on the low energy bands within the dashed-line box. bands of BLG strikingly change. First, the built-in dipole moment in the heterostructure $[22,27]$ separates the conduction and valence bands, inducing a band gap for a given spin polarization. Next, the states exhibit a giant spin-orbit coupling induced from the TMDC layer. This coupling is opposite in $K$ and $K^{\prime}$ points, as a consequence of time reversal symmetry of the spin-orbit interaction. Finally, the electronic states of BLG become magnetic, manifested by an exchange splitting, equal in $K$ and $K^{\prime}$, coming from the ferromagnetic CGT. It is the fascinating spectral separation of the spin-orbit coupling and exchange in the BLG which allows for their swapping.

The three aforementioned effects are nicely seen in Fig. 3 which shows a zoom to the low-energy band structure of the stack at both $K$ and $K^{\prime}$. Perhaps the most striking feature of the band structure is the difference in the energy dispersion at $K$ and $K^{\prime}$, which ultimately comes from the interplay between the induced spin-orbit and exchange couplings in BLG. While the valence and conduction bands partially overlap at $K$, there is a local band gap at $K^{\prime}$. The dipole moment of the heterostructure is too weak to open a global (over the whole Brillouin zone) band gap. The spin splittings are $2-8 \mathrm{meV}$, which is experimentally significant.

An applied external electric field increases the band gap of the doubly proximitized BLG and makes explicit the effects of the TMDC and CGT layers. This is demonstrated in Fig. 3, which shows both the DFT results (for fields $-0.5,0$, and $0.4 \mathrm{~V} / \mathrm{nm}$ ) and model calculations (for fields, $-1,-0.5,0,0.4$, and $1 \mathrm{~V} / \mathrm{nm})$ using an effective Hamiltonian introduced in the next section. Let us first look at negative electric fields, say $-1 \mathrm{~V} / \mathrm{nm}$, which point down, towards CGT. The conduction bands are split by about $8 \mathrm{meV}$, having identical spin polarizations at $K$ and $K^{\prime}$, which means that this splitting is due to exchange coupling: the conduction bands are affected by the ferromagnetic CGT and conduction electrons will exhibit transport properties of magnetic conductors. On the other hand, the valence bands are split less, by about $2 \mathrm{meV}$. More important, the spin polarizations of the two bands are opposite at $K$ and $K^{\prime}$, signaling time-reversal symmetry: the valence bands experience spin-orbit coupling from $\mathrm{WS}_{2}$. This makes sense. At negative electric fields electrons in the upper layer of BLG have a lower energy, and form the valence band. These electrons are affected by the TMDC layer, which gives them the strong spin-orbit coupling character. We will see in the next section that the spin-orbit coupling is of the valley Zeeman type, which is characteristic for graphene proximitized by a TMDC. Similarly, electron orbitals in the lower layer of BLG have a higher energy, forming the conduction band, which is magnetic due to the presence of CGT. If the applied electric field points up, towards the TMDC layer, the situation is reversed and the spin characters of the valence and conduction bands are swapped, see Fig. 3. 


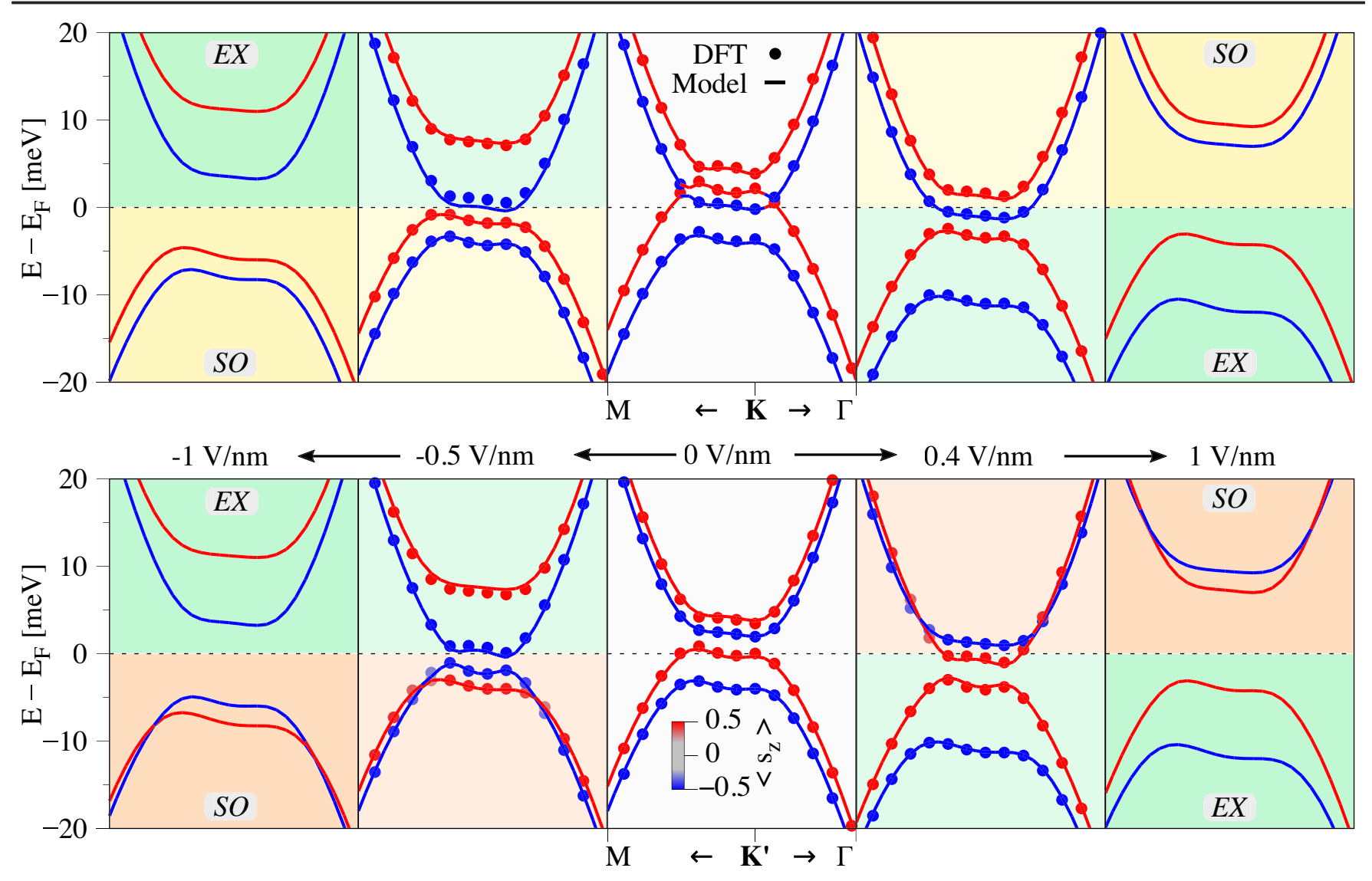

FIG. 3. Low energy dispersion curves around $\mathrm{K}$ (top) and $\mathrm{K}^{\prime}$ (bottom) for the $\mathrm{WS}_{2} / \mathrm{BLG} / \mathrm{CGT}$ heterostructure. The color of the curves corresponds to the $s_{z}$ spin expectation value: spin up is red and spin down is blue. The yellowish and reddish backgrounds indicate that the bands are split by spin-orbit coupling (SO): the yellowish is for down-up (blue-red) and reddish for up-down (red-blue) spin ordering along increasing energy. The greenish background is for exchange coupling (EX) whose ordering is always up-down, fixed by the magnetization of the CGT layer. From left to right the transverse electric field is tuned from -1 to $1 \mathrm{~V} / \mathrm{nm}$. For electric fields of $-0.5,0$, and $0.4 \mathrm{~V} / \mathrm{nm}$ both model (solid lines) and DFT data (symbols) are plotted. For electric fields of $\pm 1 \mathrm{~V} / \mathrm{nm}$ we use the model parameters with extrapolated values for $V$, assuming a linear dependence on the field. Parameters $V=7.1,3.2,-0.5,-3.6$, $-8.1 \mathrm{meV}$ correspond to the field values of $-1,-0.5,0,0.4,1 \mathrm{~V} / \mathrm{nm}$, respectively.

We have thus demonstrated the swapping of exchange and spin-orbit couplings: at a fixed chemical potential (doping level), the electrons exhibit either exchange or spin-orbit coupling, not both. Which coupling is realized depends on the gate. The same would work with few-layer TMDCs and ferromagnetic semiconductors encapsulating BLG, since the proximity effect is sensitive to the interfacial layers only [52].

Model Hamiltonian.-To describe the low-energy states of our heterostructure we employ a Hamiltonian for BLG in the presence of a transverse electric field [37],

$$
\mathcal{H}=\mathcal{H}_{\text {orb }}+\mathcal{H}_{\text {soc }}+\mathcal{H}_{R}+\mathcal{H}_{\text {ex }}+E_{D}
$$

It comprises orbital (orb), intrinsic spin-orbit (soc), Rashba $(R)$, and exchange (ex) terms. We also include the Diracpoint energy $E_{D}$ shift. Below we specify the individual Hamiltonian terms using pseudospin notation. A matrix form of $\mathcal{H}$ is given in the Supplemental Material [36].
The orbital physics is captured by

$$
\begin{aligned}
\mathcal{H}_{\mathrm{orb}}= & -\frac{\sqrt{3} \gamma_{0} a}{2} \mu_{0} \otimes\left(\tau k_{x} \sigma_{x}+k_{y} \sigma_{y}\right) \otimes s_{0} \\
& +\frac{\gamma_{1}}{2}\left(\mu_{x} \otimes \sigma_{x}-\mu_{y} \otimes \sigma_{y}\right) \otimes s_{0} \\
& -\frac{\sqrt{3} \gamma_{3} a}{4} \mu_{x} \otimes\left(\tau k_{x} \sigma_{x}-k_{y} \sigma_{y}\right) \otimes s_{0} \\
& -\frac{\sqrt{3} \gamma_{3} a}{4} \mu_{y} \otimes\left(\tau k_{x} \sigma_{y}+k_{y} \sigma_{x}\right) \otimes s_{0} \\
& -\frac{\sqrt{3} \gamma_{4} a}{2}\left(\tau k_{x} \mu_{x}-k_{y} \mu_{y}\right) \otimes \sigma_{0} \otimes s_{0} \\
& +V \mu_{z} \otimes \sigma_{0} \otimes s_{0} \\
& +\Delta\left(\mu_{+} \otimes \sigma_{+}+\mu_{-} \otimes \sigma_{-}\right) \otimes s_{0},
\end{aligned}
$$

where we denote the graphene lattice constant $a$ and the wave vectors $k_{x}$ and $k_{y}$, measured from $\pm \mathrm{K}$ for the valley 
index $\tau= \pm 1$. The Pauli matrices $\mu_{i}, \sigma_{i}$, and $s_{i}$, represent layer, pseudospin, and spin, with $i=\{0, x, y, z\}$. We also define $\mu_{ \pm}=\frac{1}{2}\left(\mu_{z} \pm \mu_{0}\right)$ and $\sigma_{ \pm}=\frac{1}{2}\left(\sigma_{z} \pm \sigma_{0}\right)$ to shorten notation. Parameters $\gamma_{j}, j=\{0,1,3,4\}$, denote intra- and interlayer hoppings of the BLG. Transverse displacement field is introduced by voltage $V$ for the lower, and $-V$ for the upper layer of BLG. Finally, $\Delta$ is the asymmetry in the energy shift of the bonding and antibonding states.

The intrinsic spin-orbit coupling term, while also present in a free-standing BLG, is strongly renormalized by the proximity to the TMDC. This effect is described by

$$
\begin{aligned}
\mathcal{H}_{\mathrm{soc}}= & \mu_{+} \otimes \tau\left(\lambda_{I}^{A 1} \sigma_{+}+\lambda_{I}^{B 1} \sigma_{-}\right) \otimes s_{z} \\
& -\mu_{-} \otimes \tau\left(\lambda_{I}^{A 2} \sigma_{+}+\lambda_{I}^{B 2} \sigma_{-}\right) \otimes s_{z},
\end{aligned}
$$

with parameters $\lambda_{I}$ denoting the proximity spin-orbit coupling of the corresponding layer $(1,2)$ and sublattice $(A, B)$ atom. Because of the short-rangeness of the proximity effect only the upper layer is affected, so that only parameters $\lambda_{I}^{A 2}$ and $\lambda_{I}^{B 2}$ are significant (on the meV scale). The Rashba coupling can emerge due to the breaking of the space inversion symmetry in the heterostructure and the applied electric field. This term has the form

$$
\mathcal{H}_{R}=\frac{1}{2}\left(\lambda_{0} \mu_{z}+2 \lambda_{R} \mu_{0}\right) \otimes\left(\tau \sigma_{x} \otimes s_{y}-\sigma_{y} \otimes s_{x}\right),
$$

where $\lambda_{0}$ describes the local (intrinsic) breaking of space inversion due to the presence of the other layer in BLG. The resulting spin-orbit fields are opposite in the two layers, giving no net effect on the spin-orbit splitting. The global breaking of space inversion due to the heterostructure and the electric field is accounted for by the proper Rashba parameter $\lambda_{R}$. For a more detailed description of the model and parameters, we refer the reader to Ref. [37]. Finally, the magnetic proximity effect induces exchange coupling in BLG, which has the standard form

$$
\begin{aligned}
\mathcal{H}_{\mathrm{ex}}= & \mu_{+} \otimes\left(-\lambda_{\mathrm{ex}}^{A 1} \sigma_{+}+\lambda_{\mathrm{ex}}^{B 1} \sigma_{-}\right) \otimes s_{z} \\
& -\mu_{-} \otimes\left(-\lambda_{\mathrm{ex}}^{A 2} \sigma_{+}+\lambda_{\mathrm{ex}}^{B 2} \sigma_{-}\right) \otimes s_{z}
\end{aligned}
$$

where parameters $\lambda_{\mathrm{ex}}$ represent the proximity exchange for the individual sublattices and layers.

The spectrum of the effective Hamiltonian $\mathcal{H}$ is fitted to the DFT-obtained low-energy dispersion of doubly proximitized BLG at zero applied electric field shown in Fig. 2.
First, the orbital parameters $\gamma_{j}$ from $\mathcal{H}_{\text {orb }}$ are obtained, as well as the built-in bias $V$, staggered potential $\Delta$, and the Dirac energy $E_{D}$. In the second step we analyze the fine structure of the spectrum and extract proximity induced spin-orbit and exchange parameters. The induced spin-orbit coupling in the upper layer is of the valley-Zeeman type, with opposite parameters on the two atoms of the sublattice: $\lambda_{I}^{A 2}=-\lambda_{I}^{B 2} \approx 1.1 \mathrm{meV}$. The atoms of the lower BLG layer experience strong proximity exchange coupling due to the adjacent CGT, $\lambda_{\mathrm{ex}}^{A 1}=\lambda_{\mathrm{ex}}^{B 1} \approx-3.9 \mathrm{meV}$. As for the Rashba coupling, it is negligible (on the meV scale), which is consistent with our finding that the spin polarization of the considered bands is predominantly out of plane. The fitted parameters are summarized in Table I; parameters not presented there were found to be negligible and set to zero. With such a minimal set of parameters the agreement of the effective model and the DFT is excellent, see Fig. 3.

The fitted parameters demonstrate the message that one layer of BLG experiences giant (on the meV scale) spin-orbit coupling, while the other layer giant exchange coupling, at zero electric field. The only parameter that significantly changes when an electric field is applied is $V$. We therefore change $V$ in our model Hamiltonian (and keep other parameters as fitted to the zero-field DFT results) to see how the spectra develop. The results are presented in Fig. 3. Their qualitative interpretation was already given above. Here we only note that the consistency of this procedure (changing only $V$ as the electric field is applied) is checked by performing DFT simulations for two electric fields, -0.5 and $0.4 \mathrm{~V} / \mathrm{nm}$; for $\pm 1 \mathrm{~V} / \mathrm{nm}$ we use $V$ assuming its linear dependence on the applied electric field. The agreement with the model calculations, seen in Fig. 3, gives us full confidence in our approach. More detailed fit results can be found in the Supplemental Material [36], where we also provide results for the individual BLG/CGT and $\mathrm{WS}_{2} / \mathrm{BLG}$ subsystems, to further validate the robustness of the model.

For an experimental realization of the swapping effect it is preferred to dope BLG with holes, to prevent spilling out the conduction-band carriers into the CGT (the Dirac point is about $100 \mathrm{meV}$ below the conduction band of CGT, see Fig. 2), upon application of a field. However, we do not expect such a spill-out for conduction-electrons to happen at fields lower than $0.5 \mathrm{~V} / \mathrm{nm}$ [27].

\begin{tabular}{|c|c|c|c|c|c|c|c|c|c|c|c|}
\hline$\gamma_{0}$ & $\gamma_{1}$ & $\gamma_{3}$ & $\gamma_{4}$ & $V$ & $\Delta$ & $\lambda_{I}^{\mathrm{A} 2}$ & $\lambda_{I}^{B 2}$ & $\lambda_{\mathrm{ex}}^{A 1}$ & $\lambda_{\mathrm{ex}}^{B 1}$ & $E_{D}$ & Dipole \\
\hline 2.432 & 0.365 & -0.273 & -0.164 & -0.474 & 8.854 & 1.132 & -1.132 & -3.874 & -3.874 & 0.348 & 0.398 \\
\hline
\end{tabular}

TABLE I. Parameters of the model Hamiltonian $\mathcal{H}$, Eq. (1), fitted to the DFT low-energy dispersion data for the CGT/BLG/WS 2 heterostructure at zero applied electric field. Parameters $\gamma$ are in $\mathrm{eV}$, others in meV. The dipole is given in debye. Unspecified model parameters are zero. Based on the fit parameters for the individual BLG/CGT and $\mathrm{WS}_{2} / \mathrm{BLG}$ subsystems given in the Supplemental Material, we assumed $\lambda_{I}^{\mathrm{A} 2}=-\lambda_{I}^{B 2}$ and $\lambda_{\mathrm{ex}}^{A 1}=\lambda_{\mathrm{ex}}^{B 1}$ here for the fit. 
Summary.-Doubly proximitized BLG offers a unique platform for investigating fundamental spin physics and designing multifunctional spintronics applications. Using realistic DFT simulations and phenomenological modeling we demonstrate swapping the two most important spin interactions-exchange and spin-orbit couplings-in a $\mathrm{WS}_{2} / \mathrm{BLG} / \mathrm{CGT}$ multilayer. The swapping also means turning the time-reversal symmetry on and off, on demand, in the electronic states at a given doping. Since the effect is robust, we expect a variety of swapping phenomena if the bilayer sandwich comprises antiferromagnets, ferroelectricity, ferroelectrics, topological insulators, or superconductors.

This work was funded by the Deutsche Forschungsgemeinschaft (DFG, German Research Foundation) SFB 1277 (Project-ID 314695032), the European Unions Horizon 2020 research and innovation program under Grant No. 785219, DFG SPP 1666, and by the VEGA 1/0105/20 and Internal Research Grant System VVGS-2019-1227.

*klaus.zollner@physik.uni-regensburg.de

[1] I. Zutic, J. Fabian, and S. Das Sarma, Spintronics: Fundamentals and applications, Rev. Mod. Phys. 76, 323 (2004).

[2] W. Han, R. K. Kawakami, M. Gmitra, and J. Fabian, Graphene spintronics, Nat. Nanotechnol. 9, 794 (2014).

[3] A. Avsar, H. Ochoa, F. Guinea, B. Özyilmaz, B. J. van Wees, and I. J. Vera-Marun, Colloquium: Spintronics in graphene and other two-dimensional materials, Rev. Mod. Phys. 92, 021003 (2020).

[4] S. Datta and B. Das, Electronic analog of the electrooptic modulator, Appl. Phys. Lett. 56, 665 (1990).

[5] J. Schliemann, J. C. Egues, and D. Loss, Nonballistic SpinField-Effect Transistor, Phys. Rev. Lett. 90, 146801 (2003).

[6] J. Wunderlich, B.-G. Park, A. C. Irvine, L. P. Zarbo, E. Rozkotova, P. Nemec, V. Novak, J. Sinova, and T. Jungwirth, Spin Hall effect transistor, Science 330, 1801 (2010).

[7] C. Betthausen, T. Dollinger, H. Saarikoski, V. Kolkovsky, G. Karczewski, T. Wojtowicz, K. Richter, and D. Weiss, Spintransistor action via tunable Landau-Zener transitions, Science 337, 324 (2012).

[8] P. Chuang et al., All-electric all-semiconductor spin fieldeffect transistors, Nat. Nanotechnol. 10, 35 (2015).

[9] Z. Wang, D.-K. Ki, H. Chen, H. Berger, A. H. MacDonald, and A.F. Morpurgo, Strong interface-induced spin-orbit interaction in graphene on $\mathrm{WS}_{2}$, Nat. Commun. 6, 8339 (2015).

[10] A. Avsar, D. Unuchek, J. Liu, O. L. Sanchez, K. Watanabe, T. Taniguchi, B. Özyilmaz, and A. Kis, Optospintronics in graphene via proximity coupling, ACS Nano 11, 11678 (2017).

[11] Y. K. Luo, J. Xu, T. Zhu, G. Wu, E. J. McCormick, W. Zhan, M. R. Neupane, and R. K. Kawakami, Opto-valleytronic spin injection in monolayer $\mathrm{MoS}_{2} /$ few-layer graphene hybrid spin valves, Nano Lett. 17, 3877 (2017).
[12] D. Zhong et al., Layer-resolved magnetic proximity effect in van der Waals heterostructures, Nat. Nanotechnol. 15, 187 (2020).

[13] B. Karpiak et al., Magnetic proximity in a van der Waals heterostructure of magnetic insulator and graphene, 2D Mater. 7, 015026 (2019).

[14] T. S. Ghiasi, J. Ingla-Aynés, A. A. Kaverzin, and B. J. Van Wees, Large proximity-induced spin lifetime anisotropy in transition-metal dichalcogenide/graphene heterostructures, Nano Lett. 17, 7528 (2017).

[15] T. S. Ghiasi, A. A. Kaverzin, P. J. Blah, and B. J. van Wees, Charge-to-spin conversion by the Rashba-Edelstein effect in two-dimensional van der Waals heterostructures up to room temperature, Nano Lett. 19, 5959 (2019).

[16] D. Ghazaryan et al., Magnon-assisted tunnelling in van der Waals heterostructures based on $\mathrm{CrBr}_{3}$, Nat. Electron. 1, 344 (2018).

[17] C. Safeer, J. Ingla-Aynés, F. Herling, J. H. Garcia, M. Vila, N. Ontoso, M. Reyes Calvo, S. Roche, L. E. Hueso, and F. Casanova, Room-temperature spin Hall effect in graphene $/ \mathrm{MoS}_{2}$ van der Waals heterostructures, Nano Lett. 19, 1074 (2019).

[18] I. Zutic, A. Matos-Abiague, B. Scharf, H. Dery, and K. Belashchenko, Proximitized materials, Mater. Today 22, 85 (2019).

[19] L. A. Benitez, W. S. Torres, J. F. Sierra, M. Timmermans, J. H. Garcia, S. Roche, M. V. Costache, and S. O. Valenzuela, Tunable room-temperature spin galvanic and spin Hall effects in van der Waals heterostructures, Nat. Mater. 19, 170 (2020).

[20] N. Cortés, O. Ávalos-Ovando, L. Rosales, P. A. Orellana, and S. E. Ulloa, Tunable Spin-Polarized Edge Currents in Proximitized Transition Metal Dichalcogenides, Phys. Rev. Lett. 122, 086401 (2019).

[21] S. Zihlmann, A. W. Cummings, J. H. Garcia, M. Kedves, K. Watanabe, T. Taniguchi, C. Schönenberger, and P. Makk, Large spin relaxation anisotropy and valley-Zeeman spinorbit coupling in $\mathrm{WSe}_{2} /$ graphene/ $h$-BN heterostructures, Phys. Rev. B 97, 075434 (2018).

[22] M. Gmitra and J. Fabian, Proximity Effects in Bilayer Graphene on Monolayer $\mathrm{WSe}_{2}$ : Field-Effect Spin Valley Locking, Spin-Orbit Valve, and Spin Transistor, Phys. Rev. Lett. 119, 146401 (2017).

[23] J. Y. Khoo, A. F. Morpurgo, and L. Levitov, On-demand spin-orbit interaction from which-layer tunability in bilayer graphene, Nano Lett. 17, 7003 (2017).

[24] J. O. Island et al., Spin-orbit-driven band inversion in bilayer graphene by the van der Waals proximity effect, Nature (London) 571, 85 (2019).

[25] P. Michetti, P. Recher, and G. Iannaccone, Electric field control of spin rotation in bilayer graphene, Nano Lett. 10, 4463 (2010).

[26] C. Cardoso, D. Soriano, N. A. García-Martínez, and J. Fernández-Rossier, Van der Waals Spin Valves, Phys. Rev. Lett. 121, 067701 (2018).

[27] K. Zollner, M. Gmitra, and J. Fabian, Electrically tunable exchange splitting in bilayer graphene on monolayer $\mathrm{Cr}_{2} \mathrm{X}_{2} \mathrm{Te}_{6}$ with $\mathrm{X}=\mathrm{Ge}, \mathrm{Si}$, and $\mathrm{Sn}$, New J. Phys. 20, 073007 (2018). 
[28] M. Alghamdi, M. Lohmann, J. Li, P. R. Jothi, Q. Shao, M. Aldosary, T. Su, B. P. T. Fokwa, and J. Shi, Highly efficient spin-orbit torque and switching of layered ferromagnet $\mathrm{Fe}_{3} \mathrm{GeTe}_{2}$, Nano Lett. 19, 4400 (2019).

[29] K. Dolui, M. D. Petrović, K. Zollner, P. Plecháč, J. Fabian, and B. K. Nikolić, Proximity spin-orbit torque on a twodimensional magnet within van der Waals heterostructure: Current-driven antiferromagnet-to-ferromagnet reversible nonequilibrium phase transition in bilayer $\mathrm{CrI}_{3}$, Nano Lett. 20, 2288 (2020).

[30] D. MacNeill, G. M. Stiehl, M. H. D. Guimaraes, R. A. Buhrman, J. Park, and D. C. Ralph, Control of spin-orbit torques through crystal symmetry in $\mathrm{WTe}_{2} /$ ferromagnet bilayers, Nat. Phys. 13, 300 (2017).

[31] C. L. Kane and E. J. Mele, Quantum Spin Hall Effect in Graphene, Phys. Rev. Lett. 95, 226801 (2005).

[32] A. W. Cummings, J. H. Garcia, J. Fabian, and S. Roche, Giant Spin Lifetime Anisotropy in Graphene Induced by Proximity Effects, Phys. Rev. Lett. 119, 206601 (2017).

[33] S. Omar, B. N. Madhushankar, and B. J. van Wees, Large spin-relaxation anisotropy in bilayer-graphene/ $\mathrm{WS}_{2}$ heterostructures, Phys. Rev. B 100, 155415 (2019).

[34] L. A. Benitez, J. F. Sierra, W. S. Torres, A. Arrighi, F. Bonell, M. V. Costache, and S. O. Valenzuela, Strongly anisotropic spin relaxation in graphene-transition metal dichalcogenide heterostructures at room temperature, Nat. Phys. 14, 303 (2018).

[35] H. X. Yang, A. Hallal, D. Terrade, X. Waintal, S. Roche, and M. Chshiev, Proximity Effects Induced in Graphene by Magnetic Insulators: First-Principles Calculations on Spin Filtering and Exchange-Splitting Gaps, Phys. Rev. Lett. 110, 046603 (2013).

[36] See Supplemental Material at http://link.aps.org/ supplemental/10.1103/PhysRevLett.125.196402, including Refs. [22,24,27,37-51], for the computational details and results for the individual BLG/CGT and $\mathrm{WS}_{2} / \mathrm{BLG}$ heterostructures. In addition, we show an animation about the electric field evolution of the low energy bands of doubly proximitized BLG.

[37] S. Konschuh, M. Gmitra, D. Kochan, and J. Fabian, Theory of spin-orbit coupling in bilayer graphene, Phys. Rev. B 85, 115423 (2012).

[38] Y. Baskin and L. Meyer, Lattice constants of graphite at low temperatures, Phys. Rev. 100, 544 (1955).
[39] G. Kresse and D. Joubert, From ultrasoft pseudopotentials to the projector augmented-wave method, Phys. Rev. B 59, 1758 (1999).

[40] J. P. Perdew, K. Burke, and M. Ernzerhof, Generalized Gradient Approximation Made Simple, Phys. Rev. Lett. 77, 3865 (1996).

[41] S. Grimme, Semiempirical GGA-type density functional constructed with a long-range dispersion correction, J. Comput. Chem. 27, 1787 (2006).

[42] V. Barone, M. Casarin, D. Forrer, M. Pavone, M. Sambi, and A. Vittadini, Role and effective treatment of dispersive forces in materials: Polyethylene and graphite crystals as test cases, J. Comput. Chem. 30, 934 (2009).

[43] L. Bengtsson, Dipole correction for surface supercell calculations, Phys. Rev. B 59, 12301 (1999).

[44] V. Carteaux, D. Brunet, G. Ouvrard, and G. Andre, Crystallographic, magnetic and electronic structures of a new layered ferromagnetic compound $\mathrm{Cr}_{2} \mathrm{Ge}_{2} \mathrm{Te}_{6}$, J. Phys. Condens. Matter 7, 69 (1995).

[45] C. Gong et al., Discovery of intrinsic ferromagnetism in two-dimensional van der Waals crystals, Nature (London) 546, 265 (2017).

[46] W. J. Schutte, J. L. De Boer, and F. Jellinek, Crystal structures of tungsten disulfide and diselenide, J. Solid State Chem. 70, 207 (1987).

[47] P. Hohenberg and W. Kohn, Inhomogeneous electron gas, Phys. Rev. 136, B864 (1964).

[48] P. Giannozzi et al., Quantum Espresso: A modular and open-source software project for quantum simulations of materials, J. Phys. Condens. Matter 21, 395502 (2009).

[49] K. Wang, T. Hu, F. Jia, G. Zhao, Y. Liu, I. V. Solovyev, A. P. Pyatakov, A. K. Zvezdin, and W. Ren, Magnetic and Electronic Properties of $\mathrm{Cr}_{2} \mathrm{Ge}_{2} \mathrm{Te}_{6}$ Monolayer by Strain and Electric-Field Engineering, Appl. Phys. Lett. 114, 092405 (2019).

[50] X. Chen, J. Qi, and D. Shi, Strain-engineering of magnetic coupling in two-dimensional magnetic semiconductor $\mathrm{CrSiTe}_{3}$ : Competition of direct exchange interaction and superexchange interaction, Phys. Lett. A 379, 60 (2015).

[51] X. Li and J. Yang, $\mathrm{CrXTe}_{3}(\mathrm{X}=\mathrm{Si}, \mathrm{Ge})$ nanosheets: Two dimensional intrinsic ferromagnetic semiconductors, J. Mater. Chem. C 2, 7071 (2014).

[52] K. Zollner, P. E. Faria Junior, and J. Fabian, Proximity exchange effects in $\mathrm{MoSe}_{2}$ and $\mathrm{WSe}_{2}$ heterostructures with $\mathrm{CrI}_{3}$ : Twist angle, layer, and gate dependence, Phys. Rev. B 100, 085128 (2019). 